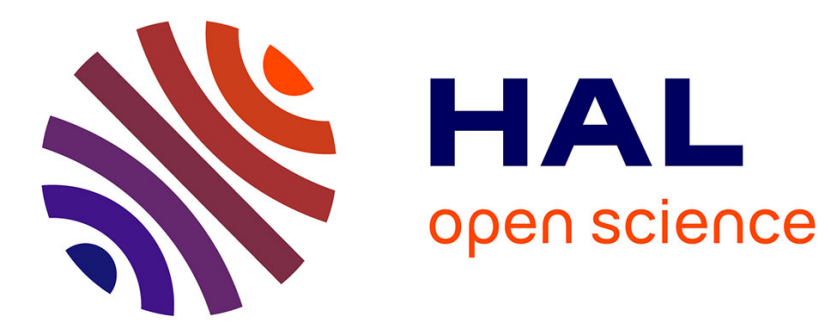

\title{
Evaluation of the Immunoquick +4 malaria rapid diagnostic test in a non-endemic setting
}

D. P. J. Dijk, P. Gillet, E. Vlieghe, L. Cnops, M. Esbroeck, J. Jacobs

\section{To cite this version:}

D. P. J. Dijk, P. Gillet, E. Vlieghe, L. Cnops, M. Esbroeck, et al.. Evaluation of the Immunoquick+4 malaria rapid diagnostic test in a non-endemic setting. European Journal of Clinical Microbiology and Infectious Diseases, 2010, 29 (5), pp.577-583. 10.1007/s10096-010-0898-y . hal-00577008

\section{HAL Id: hal-00577008 \\ https://hal.science/hal-00577008}

Submitted on 16 Mar 2011

HAL is a multi-disciplinary open access archive for the deposit and dissemination of scientific research documents, whether they are published or not. The documents may come from teaching and research institutions in France or abroad, or from public or private research centers.
L'archive ouverte pluridisciplinaire HAL, est destinée au dépôt et à la diffusion de documents scientifiques de niveau recherche, publiés ou non, émanant des établissements d'enseignement et de recherche français ou étrangers, des laboratoires publics ou privés. 


\title{
Evaluation of the Immunoquick +4 malaria rapid diagnostic test in a non-endemic setting
}

\author{
D. P. J. van Dijk • P. Gillet • E. Vlieghe • L. Cnops • \\ M. Van Esbroeck • J. Jacobs
}

Received: 22 September 2009 / Accepted: 13 February 2010/Published online: 16 March 2010

(C) Springer-Verlag 2010

\begin{abstract}
The aim of this retrospective study was to evaluate the Immunoquick+4 (BioSynex, Strasbourg, France), a three-band malaria rapid diagnostic test (MRDT) targeting histidine-rich protein-2 (HRP-2) and pan Plasmodium-specific parasite lactate dehydrogenase, in a non-endemic reference setting. Stored whole-blood samples $(n=613)$ from international travellers suspected of malaria were used, with microscopy corrected by polymerase chain reaction (PCR) as the reference method. Samples infected by P. falciparum $(n=323)$, P. vivax $(n=97)$, P. ovale $(n=73)$ and P. malariae $(n=25)$ were selected, as well as 95 malaria-negative samples. The overall sensitivities
\end{abstract}

D. P. J. van Dijk · J. Jacobs

Medical Microbiology, Faculty of Health,

Medicine and Life Sciences (FHML), Maastricht University,

Maastricht, The Netherlands

D. P. J. van Dijk

e-mail: D.vanDijk@student.maastrichtuniversity.nl

J. Jacobs

e-mail: jjacobs@itg.be

P. Gillet $(\varangle) \cdot$ E. Vlieghe $\cdot$ L. Cnops $\cdot$ M. Van Esbroeck

J. Jacobs

Department of Clinical Sciences,

Unit of Tropical Laboratory Medicine,

Institute of Tropical Medicine (ITM),

Nationalestraat 155,

2000 Antwerp, Belgium

e-mail: pgillet@itg.be

E. Vlieghe

e-mail: evlieghe@itg.be

L. Cnops

e-mail: lcnops@itg.be

M. Van Esbroeck

e-mail: mvesbroeck@itg.be of the Immunoquick +4 for the diagnosis of $P$. falciparum, $P$. vivax, $P$. malariae and $P$. ovale were $88.9,75.3,56.0$ and $19.2 \%$, respectively. Sensitivity was significantly related to parasite density for $P$. falciparum $(93.6 \%$ versus $71.4 \%$ at parasite densities $>100 / \mu 1$ and $\leq 100 / \mu 1$, respectively) and P. vivax $(86.8 \%$ versus $48.3 \%$ at parasite densities $>500 / \mu 1$ and $\leq 500 / \mu 1$, respectively). The Immunoquick +4 showed good reproducibility and reliability for both test results and line intensities. The Immunoquick +4 performed well for the detection of P. falciparum and P. vivax.

\section{Background}

For the diagnosis of malaria, microscopy is considered as the reference method, but expert microscopy may be lacking in both endemic and non-endemic settings. In resource-poor endemic settings, there may be problems related to equipment, expertise and workload, whereas in non-endemic settings in industrialised countries, there may be a lack of routine among the laboratory staff, resulting in low expertise [1]. In these circumstances, the use of malaria rapid diagnostic tests (MRDTs) can be valuable in the diagnosis of malaria $[1,2]$. MRDTs detect antigens specific to one or more of the Plasmodium species. The initially developed tests were two-band tests, including a control line and a $P$. falciparum-specific line targeting histidine-rich protein-2 (HRP-2) or P. falciparum-specific parasite lactate dehydrogenase (Pf-pLDH). Later developed so-called three-band MRDTs also detect antigens common to the four Plasmodium species, such as aldolase or the pan Plasmodium-specific pLDH (pan-pLDH). The Immunoquick malaria +4 (BioSynex, Strasbourg, France), further referred to as Immunoquick +4 , is a three-band MRDT targeting HRP-2 and pan-pLDH. We evaluated the 
Immunoquick +4 for use on returned international travellers in a non-endemic reference centre.

\section{Methods}

\section{Study design}

In this retrospective study, the Immunoquick +4 was evaluated against a collection of stored samples obtained from international travellers. Tests were carried out in the reference laboratory of the Institute of Tropical Medicine (ITM), Antwerp, Belgium.

\section{Patients and samples}

Samples were selected from a collection of EDTA blood samples stored at $-70^{\circ} \mathrm{C}$. They were obtained from patients attending the travel clinic at the ITM. Included patients were international travellers or immigrants returning from visits to their native countries. In addition, samples sent by Belgian laboratories to the ITM in the scope of the national reference function were included. All patients had symptoms suspect for malaria (e.g. fever, headache, nausea). All positive samples collected between December 1995 to August 2008 were selected, including the four malaria species with varying parasite densities. In addition, samples from symptomatic patients without malaria parasites (negative samples) attending the ITM between December 1995 to 2008 were included. There were no exclusion criteria except a blood sample lower than $100 \mu$ l.

\section{Reference method}

Microscopy, corrected by polymerase chain reaction (PCR), was used as the reference method. Standard microscopy including the determination of parasite density was performed on thick blood films as described previously [3]. PCR analysis was performed on all samples, with a species-specific real-time PCR adapted from Rougemont et al. [4], as described previously[3].

\section{Test platform}

The Immunoquick +4 is a lateral-flow immunochromatographic MRDT in a dipstick format. In brief, $20 \mu 1$ of whole blood is deposited on the test strip, which is subsequently transferred to a plastic tube containing six drops of buffer. After 15 to $30 \mathrm{~min}$, the results can be read. Three lines are present; a control line which indicates whether the test is valid, a HRP-2 line and a pan-pLDH line. According to the manufacturer's instructions, a combination of a HRP-2 line and a pan-pLDH line indicates an infection with $P$. falciparum or a mixed infection with $P$. falciparum and one or more of the other Plasmodium species. A single HRP-2 line indicates an infection with $P$. falciparum and a single pan-pLDH line indicates an infection with one or more of the nonfalciparum species. For the evaluation, test kits of two different lot numbers were used, M4D060508 and M4D091708, with expiration dates 01/2010 and 04/2010, respectively.

\section{Test procedure}

Tests were performed according to the instructions of the manufacturer, except that a scoring system was used to assess the intensity of the test lines. As recommended by the manufacturer in the instructions for application of the test on venous whole blood, we used a transfer pipette of $20 \mu \mathrm{l}$ (Finnpipette, Helsinki, Finland) to apply blood to the test strip. In case the control line did not appear, the result was interpreted as invalid and the test was repeated. In order to score test line intensities, we used a scoring system of five categories: none (no line visible), faint (barely visible line), weak (paler than the control line), medium (equal to the control line) or strong (stronger than the control line) [3]. Readings were performed with daylight assisted by a standard halogen lamp. To homogenise the results, all samples were read after 20 to $30 \mathrm{~min}$, as recommended by the manufacturer for low positive samples. Readings were carried out by three subsequent observers, of whom the one who performed the test procedure invariably was the first. Observers were blinded to the results of microscopy, PCR and to each others' readings. The results of the readings considered were based on consensus agreement, which means that a positive result was defined as a result read positive by at least two out of three different observers. When there was no consensus, the result of the first reader was considered. Inter-reader reliabilities were assessed for the test results, expressed as positive and negative readings, as well as for the intensity readings. To assess reproducibility, we tested a panel of 16 samples with different parasite densities (including four $P$. falciparum samples, four $P$. vivax samples, four $P$. ovale samples and four $P$. malariae samples) on five successive occasions.

\section{Statistical analysis}

Samples infected with $P$. falciparum and the nonfalciparum species were considered separately. For the detection of $P$. falciparum, any test that generated a HRP-2 line was considered positive. Any test that did not generate a HRP-2 line was considered negative. For the detection of the non-falciparum species, any test that generated a 
unique pan-pLDH line was considered positive. Any test that did not generate a test line or showed a HRP-2 line was considered negative. Samples that generated a wrong test line (e.g. a $P$. falciparum sample generating only a pan-pLDH line) were considered as species mismatch and assigned to the false-positives or false-negatives, depending on the situation. Samples with pure gametocytaemia were included among the positive $P$. falciparum samples. Sensitivity and specificity were calculated for both $P$. falciparum and the non-falciparum species with 95\% confidence intervals (CIs) and differences were tested for significance using the Pearson Chi-square test or, in case of small sample numbers, Fisher's exact probability test. A $p$-value of $<0.05$ was considered as significant. Reliabilities for positive and negative readings and line intensities were calculated as percentage agreements for all three readers and kappa values for each pair of readers. Associations between line intensity readings and parasite densities were assessed for strength of association with Cramer's $V$ for categorical variables, using interpretative criteria published previously [3].

\section{Duration of storage}

Long-term storage of samples could affect antigen stability. To minimise the possibility that the duration of storage affects the test performance, we compared the sensitivities of samples obtained between 1995 to 2000 with those obtained between 2001 to 2008 .

\section{Ease of use}

Three experienced laboratory technicians scored the ease of use of the Immunoquick +4 test and the clarity of the manufacturer's instructions with a standardised list [3].

\section{Ethical review}

The study was reviewed and approved by the Institutional Review Board of the ITM and by the Ethical Committee of Antwerp University, Belgium.

\section{Results}

Sample collection

A total of 613 samples were selected, of which 83 samples were sent by Belgian laboratories to the ITM for second opinion and confirmation. According to microscopy and after correction by PCR analysis, 323 of these samples were positive for $P$. falciparum, 97 for $P$. vivax, 73 for $P$. ovale and 25 for $P$. malariae. The results of microscopy were corrected in 11 out of the $518(2.1 \%)$ positive samples.
These corrections were uniquely related to $P$. vivax $-P$. ovale mismatches: in the final collection, 4/97 (4.1\%) $P$. vivax and $7 / 73(9.5 \%) P$. ovale samples had originally been identified as $P$. ovale and $P$. vivax, respectively. In addition, 95 microscopic and PCR-negative samples of symptomatic travellers were included in the panel. The majority (270/ 323) of P. falciparum infection was acquired in Africa. The samples were obtained from 613 patients, with a male: female ratio of 2.07:1 and median age of 36.5 years (range 1-84 years). Only a minority (nine patients, $1.5 \%$ ) were children less than five years old.

Invalid test results

A single sample gave an invalid result at initial testing. After $30 \mathrm{~min}$, there was no control line visible. Upon repetition, the test performed well.

\section{Sensitivity and specificity}

Table 1 lists the test results of all samples and Table 2 lists the sensitivities matched with species identification and parasite densities. For the detection of $P$. falciparum, the sensitivity was $88.9 \%$; it increased with parasite density, with values at parasite densities $>100 / \mu$ l being significantly higher as compared to those at parasite densities $\leq 100 / \mu 1$ (93.6\% versus $71.4 \%$, respectively, $p<0.001)$. For the detection of the non-falciparum species, the overall sensitivities for $P$. vivax, $P$. ovale and $P$. malariae were 75.3, 19.2 and $56.0 \%$, respectively. The difference in sensitivity between parasite densities above and below 500/ $\mu$ l was statistically significant in the case of $P$. vivax $(86.8 \%$ versus $48.3 \%, p<0.001)$. Specificities for $P$. falciparum and the non-falciparum species were 97.9\% (95.3\%-99.2\%) and 98.3\% (96.4\%-99.3\%), respectively. Eight out of the 518 (1.5\%) Plasmodium-positive samples resulted in a species mismatch (Table 1). Four P. falciparum samples reacted only with the pan-pLDH line; they had parasite densities of 180 , $210,1,123$ and $147,000 / \mu 1$, respectively, and the travel destinations were Democratic Republic of the Congo $(n=2)$, Nigeria $(n=1)$ and Ghana $(n=1)$, respectively. One $P$. vivax sample (India; $4,150 / \mu 1$ ), one $P$. ovale sample (Mali; $864 / \mu 1$ ) and one $P$. malariae sample (Nigeria; 2,200/ $\mu$ l) reacted with both HRP-2 and pan-pLDH lines. Finally, there was one P. malariae sample (Togo, $712 / \mu \mathrm{l})$ that reacted only with the HRP-2 line.

Line intensities

The line intensity readings for both HRP-2 and pan-pLDH lines were faint or weak in almost half of the true-positive readings, $136 / 287(47.4 \%)$ and 131/301 (43.5\%), respectively. Line intensity readings for HRP-2 and especially pan-pLDH 
Table 1 Test results of all samples $(n=613)$

HRP-2 = histidine-rich protein-2; pan-pLDH = pan Plasmodium-specific parasite lactate dehydrogenase

${ }^{\text {a }}$ Species mismatch

\begin{tabular}{|c|c|c|c|c|}
\hline \multirow[t]{2}{*}{ Samples } & \multicolumn{2}{|c|}{ HRP-2 line-positive } & \multicolumn{2}{|c|}{ HRP-2 line-negative } \\
\hline & $\begin{array}{l}\text { pan-pLDH } \\
\text { line-positive }\end{array}$ & $\begin{array}{l}\text { pan-pLDH } \\
\text { line-negative }\end{array}$ & $\begin{array}{l}\text { pan-pLDH } \\
\text { line-positive }\end{array}$ & $\begin{array}{l}\text { pan-pLDH } \\
\text { line-negative }\end{array}$ \\
\hline P. falciparum $(n=323)$ & 194 & 93 & $4^{\mathrm{a}}$ & 32 \\
\hline P. vivax $(n=97)$ & $1^{\mathrm{a}}$ & - & 73 & 23 \\
\hline P. ovale $(n=73)$ & $1^{\mathrm{a}}$ & - & 14 & 58 \\
\hline P. malariae $(n=25)$ & $1^{\mathrm{a}}$ & $1^{\mathrm{a}}$ & 14 & 9 \\
\hline Negative $(n=95)$ & - & 2 & 3 & 90 \\
\hline
\end{tabular}

were related to parasite densities (HRP-2: $V=0.296, p<0.001$; pan-pLDH: $V=0.620, p<0.001)$. Of interest is that, in the case of positive $P$. falciparum samples, the unique presence of a HRP-2 line almost exclusively (98.9\%, 92/93 samples)

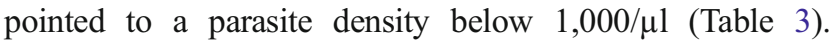
Conversely, the co-presence of both HRP-2 and pan-pLDH lines predicted parasite densities above $1,000 / \mu 1$ in $131 / 194$ $(67.5 \%)$ samples.

\section{Inter-reader reliability}

For the HRP-2 line, the inter-reader reliability for positive and negative test results was excellent, with $97.2 \%$ agreement among the three readers and kappa values between the three pairs of readers of $0.97,0.96$ and 0.95 , respectively. For the pan-pLDH line, there was $91.2 \%$ overall agreement and the kappa values were $0.91,0.88$ and 0.86. For the HRP-2 line intensity readings, the overall agreement among the three readers was $80.6 \%$, with kappa values of $0.81,0.80$ and 0.79 for the different pairs of readers. For the pan-pLDH line intensity readings, there was $76.7 \%$ overall agreement and kappa values of 0.79 , 0.78 and 0.74 .

\section{Reproducibility}

The test results (positive or negative for relevant species detection) and HRP-2 line intensity readings were reproducible. Consistent test results upon five times repetition were obtained for 10 out of 16 samples. Of the remaining six samples, five had consistent test results upon four times repetition and one sample upon three times repetition. HRP-2 line intensity readings were consistent upon five times repetition for 13 out of 16 samples. Two out of the three non-consistent samples had identical test results upon four times repetition and one sample upon three times repetition. The pan-pLDH intensity readings were consistent upon five times repetition for five out of 16 samples. Eight out of the 11 non-consistent samples had identical results upon four times repetition and three samples upon three times repetition. All but one inconsistent result for line intensity readings (13 out of 14) of the HRP-2 and pan-pLDH samples had
Table 2 Sensitivities of the Immunoquick +4 for the detection of all Plasmodium species related to parasite densities

\begin{tabular}{llll}
\hline Species & No. & $\begin{array}{l}\text { Correctly identified } \\
\text { by Immunoquick }+4\end{array}$ & $\begin{array}{l}\text { Sensitivity in \% } \\
(95 \% \text { CI })\end{array}$ \\
\hline All P. falciparum samples & 323 & 287 & $88.9(84.8-92.0)$ \\
Pure gametocytaemia & 17 & 13 & $76.5(50.0-92.2)$ \\
Parasite density $0-100 / \mu 1$ & 56 & 40 & $71.4(57.6-82.3)$ \\
Parasite density $101-1,000 / \mu 1$ & 116 & 102 & $87.9(80.3-93.0)$ \\
Parasite density $>1,000 / \mu 1$ & 134 & 132 & $98.5(94.2-99.7)$ \\
Parasite density $>100 / \mu 1$ & 250 & 234 & $93.6(89.6-96.2)$ \\
All $P$. vivax samples & 97 & 73 & $75.3(65.3-83.2)$ \\
Parasite density $0-500 / \mu 1$ & 29 & 14 & $48.3(30.0-67.1)$ \\
Parasite density $>500 / \mu 1$ & 68 & 59 & $86.8(75.9-93.4)$ \\
All $P$. ovale samples & 73 & 14 & $19.2(11.2-30.4)$ \\
Parasite density $\leq 500 / \mu 1$ & 36 & 6 & $16.7(7.0-33.5)$ \\
Parasite density $>500 / \mu 1$ & 37 & 8 & $21.6(10.4-38.7)$ \\
All P. malariae samples & 25 & 14 & $56.0(35.3-75.0)$ \\
Parasite density $\leq 500 / \mu 1$ & 9 & 4 & $44.4(15.3-77.3)$ \\
Parasite density $>500 / \mu 1$ & 16 & 10 & $62.5(35.9-83.7)$ \\
\hline
\end{tabular}


Table 3 Presence of HRP-2 and pan-pLDH lines for the Immunoquick +4 in Plasmodium falciparum samples related to parasite density

HRP-2 = histidine-rich protein-2; pan-pLDH = pan Plasmodium-specific parasite lactate dehydrogenase

\begin{tabular}{lllll}
\hline Parasite density & \multicolumn{2}{l}{ Test line(s) visible } & \\
\cline { 2 - 5 } & None & Only HRP-2 & HRP-2 and pan-pLDH & Total \\
\hline Pure gametocytaemia & 4 & 5 & 8 & 17 \\
$0-100 / \mu 1$ & 16 & 36 & 4 & 56 \\
$101-1,000 / \mu 1$ & 14 & 51 & 51 & 116 \\
$>1,000 / \mu 1$ & 2 & 1 & 131 & 134 \\
Total & 32 & 93 & 194 & 323
\end{tabular}

discordances within one category of difference in line intensity. One pan-pLDH result differed by three categories in line intensity (medium instead of negative).

\section{Duration of storage}

There was no significant difference between the test sensitivities of samples that had been stored for long versus shorter periods, for any of the four Plasmodium species. The sensitivities of samples obtained between 1995 to 2000 and samples obtained between 2001 to 2008 were, respectively, 86.9 and $89.7 \%$ for $P$. falciparum, 73.2 and $76.8 \%$ for $P$. vivax, 16.1 and $21.4 \%$ for $P$. ovale, and 66.7 and $52.4 \%$ for $P$. malariae. None of these differences were statistically significant.

\section{Ease of use}

The Immunoquick +4 was evaluated as easy to use and the instructions were scored as clear and simple to follow by all three technicians. However, because of its design (a strip soaked in a test tube), it was considered to be less practical as compared to one-step MRDTs in cassette format. Further, the clearance was not optimal, causing the test strip to remain red-coloured after completion of the test procedure.

\section{Discussion}

In this retrospective study, the performance of the Immunoquick +4 was evaluated on a large panel of stored samples obtained from international travellers. The sensitivity for the detection of $P$. falciparum was $88.9 \%$, reaching $98.5 \%$ at parasite densities above $1,000 / \mu 1$. The overall sensitivities for $P$. vivax, $P$. ovale and $P$. malariae were $75.3,19.2$ and $56 \%$, respectively. The specificity was above 97.9\%. Species mismatches occurred in $1.5 \%$ of the samples and were limited to non-falciparum species that were identified as P. falciparum. The test results were reliable and reproducible.

The present study has some limitations. For instance, we did not challenge the test with samples that are known to give false test results (such as rheumatoid factor) [5]. Further, tests were performed in a non-endemic reference centre, which tends - for reasons of resources and trained staff - to give better results compared to field settings $[6,7]$. Finally, the storage time of the present samples could affect antigen stability [6]. However, samples were not exposed to repeated thawing and freezing, and no differences in test performances were observed between samples that had been stored for long versus short periods. In addition, a prospective study revealed similar results between fresh and stored samples with regard to HRP-2 detection [8].

The Immunoquick +4 has recently been evaluated as part of the World Health Organisation (WHO) and the Foundation for Innovative New Diagnostics (FIND) Malaria RDT Evaluation Programme [9]. This evaluation showed for $P$. falciparum and $P$. vivax sensitivities of, respectively, 93.7 and $30.0 \%$ at parasite densities of $200 / \mu 1$, and sensitivities of 98.7 and $100 \%$ at parasite densities of $2,000-5,000 / \mu 1$. In addition, this study reported species mismatches in $2(1.6 \%)$ out of $120 P$. vivax samples (which showed an additional HRP-2 test line) and a single $(0.6 \%)$ false-positive reaction among 168 malaria-negative samples. The sensitivities reported by the WHO/FIND study are higher than those obtained in the present study, but the nature of samples and the parasite densities evaluated by both studies were different: for instance, the WHO/FIND study also used culture-derived samples and used samples that were diluted to parasite densities of $200 / \mu \mathrm{l}$ and $2,000-5,000 / \mu 1$. Unlike the present study, the WHO/FIND study did not include $P$. ovale and $P$. malariae samples and it assessed species mismatches only between $P$. falciparum and $P$. vivax [9].

The sensitivities of the Immunoquick +4 for the detection of $P$. falciparum and $P$. vivax are in line with those for other MRDTs in non-endemic settings. Reported sensitivities for $P$. falciparum range from 87.5 to $99.0 \%$, with one exception of $76.2 \%$ [3, 10-16]. However, the Immunoquick +4 did not reach the $95 \%$ sensitivity for parasite densities $>100 / \mu 1$ recommended by the WHO [17]. For $P$. vivax, MRDTs targeting pan-pLDH show sensitivities from $33.5 \%$ and $62.0-95.0 \%$ [3, 12, 15, 18-20], compared to $46.0-93.0 \%$ [20] for those MRDTs targeting aldolase. The higher 
sensitivities at increasing parasite densities are a well-known phenomenon, with breakpoints around 100/ $\mu 1$ (P. falciparum) and $500 / \mu 1(P . v i v a x)$, respectively $[3,10,18,20,21]$. There are only a few studies that allow the comparison of test characteristics for the detection of $P$. ovale and $P$. malariae separately. The sensitivities for the detection of $P$. ovale and $P$. malariae of the Immunoquick +4 tended to be lower compared to other MRDTs, with combined sensitivities of $36.0-95.0 \%$ for MRDTs targeting pan-pLDH $[18,20]$ and $7.0-80.0 \%$ for MRDTs targeting aldolase [20]. Previously, we demonstrated for a three-band HRP-2/pan-pLDH MRDT in a study design similar to the present one sensitivities for $P$. ovale and $P$. malariae of 76.3 and $45.2 \%$, respectively [3].

In line with other publications [3, 10, 22, 23], lines intensities were in relation with the parasite densities, but with considerable overlaps. The finding that a unique HRP-2 line was indicative for a low parasite density of $P$. falciparum $\leq 1,000 / \mu 1$ is in line with a previous observation for another MRDT based on HRP2/pan-pLDH detection [3]

Although the Immunoquick +4 was reliable and reproducible, we observed incomplete clearance of the test strip. In addition, nearly half of the test lines showed weak or faint intensities. These findings are of concern, as they can result in false-negative interpretations. Indeed, disregarding faint line intensities is one of the most common mistakes among end-users in both endemic and non-endemic settings, even when provided with adapted job aids [24-28].

The Immunoquick +4 was scored as a less practical test compared to MRDTs in cassette format. In particular, the manipulation of the strip was felt to be more difficult compared to the manipulation of a cassette. However, although not evaluated as part of the present study, we assume that the recommended transfer of the blood by applying the soak pad of the strip to the blood on the finger is easier and less error-prone than other application systems, such as loops and straws [25, 29]. On the other hand, the use of the absorption pad of the dipstick as a sampling system for capillary blood may be less accurate. Further studies at the end-user level are required to assess these assumptions.

In conclusion, the Immunoquick +4 performed well for the detection of $P$. falciparum and $P$. vivax, but moderately and poorly for the detection of $P$. malariae and $P$. ovale, respectively. The Immunoquick +4 can be a valuable adjunct to microscopy, especially in those settings where $P$. falciparum and $P$. vivax are predominant. Possible test improvements include an increase in sensitivity, especially for non-falciparum species detection, a better clearance of the test strip and an increase in test line intensities.

Acknowledgements We would like to thank the staff of the Central Laboratory of Clinical Biology for their technical support.
Authors' contributions DvD, PG and JJ designed the study protocol. MvE and EV organised prospective sample collection. DvD and PG carried out the test evaluations, LC performed PCR analysis. DvD, PG and JJ analysed and interpreted the results and drafted the manuscript. DvD performed the statistical analysis. All authors contributed to the discussion of the results and the redaction of the manuscript; they all approved the final manuscript.

\section{References}

1. Moody A (2002) Rapid diagnostic tests for malaria parasites. Clin Microbiol Rev 15:66-78

2. Hänscheid T (2003) Current strategies to avoid misdiagnosis of malaria. Clin Microbiol Infect 9:497-504

3. Van der Palen M, Gillet P, Bottieau E, Cnops L, Van Esbroeck M, Jacobs J (2009) Test characteristics of two rapid antigen detection tests (SD FK50 and SD FK60) for the diagnosis of malaria in returned travellers. Malar J 8:90

4. Rougemont M, Van Saanen M, Sahli R, Hinrikson HP, Bille J, Jaton K (2004) Detection of four Plasmodium species in blood from humans by $18 \mathrm{~S}$ rRNA gene subunit-based and speciesspecific real-time PCR assays. J Clin Microbiol 42:5636-5643

5. Iqbal J, Sher A, Rab A (2000) Plasmodium falciparum histidinerich protein 2-based immunocapture diagnostic assay for malaria: cross-reactivity with rheumatoid factors. J Clin Microbiol 38:1184-1186

6. Bell D, Peeling RW (2006) Evaluation of rapid diagnostic tests: malaria. Nat Rev Microbiol 4:S34-S38

7. Murray CK, Gasser RA Jr, Magill AJ, Miller RS (2008) Update on rapid diagnostic testing for malaria. Clin Microbiol Rev 21:97110

8. Mayxay M, Pukrittayakamee S, Chotivanich K, Looareesuwan S, White NJ (2001) Persistence of Plasmodium falciparum HRP-2 in successfully treated acute falciparum malaria. Trans R Soc Trop Med Hyg 95:179-182

9. World Health Organization (2009) Malaria rapid diagnostic test performance: executive summary. Results of WHO product testing of malaria RDTs: Round 1 (2008). Available online at: http://apps.who. int/tdr/publications/tdr-research-publications/rdt-performance/pdf/ executive-summary-malaria-RDTs.pdf. Accessed 13 June 2009

10. Farcas GA, Zhong KJ, Lovegrove FE, Graham CM, Kain KC (2003) Evaluation of the Binax NOW ${ }^{\circledR}$ ICT test versus polymerase chain reaction and microscopy for the detection of malaria in returned travelers. Am J Trop Med Hyg 69:589-592

11. Gatti S, Gramegna M, Bisoffi Z, Raglio A, Gulletta M, Klersy C, Bruno A, Maserati R, Madama S, Scaglia M (2007) A comparison of three diagnostic techniques for malaria: a rapid diagnostic test (NOW Malaria), PCR and microscopy. Ann Trop Med Parasitol 101:195-204

12. Gillet P, Bosselaers K, Cnops L, Bottieau E, Van Esbroeck M, Jacobs J (2009) Evaluation of the SD FK70 malaria Ag Plasmodium vivax rapid diagnostic test in a non-endemic setting. Malar J 8:129

13. Grobusch MP, Hänscheid T, Göbels K, Slevogt H, Zoller T, Rögler G, Teichmann D (2003) Comparison of three antigen detection tests for diagnosis and follow-up of falciparum malaria in travellers returning to Berlin, Germany. Parasitol Res 89:354357

14. Jelinek T, Schulte C, Behrens R, Grobusch MP, Coulaud JP, Bisoffi Z, Matteelli A, Clerinx J, Corachán M, Puente S, Gjørup I, Harms G, Kollaritsch H, Kotlowski A, Björkmann A, Delmont JP, Knobloch J, Nielsen LN, Cuadros J, Hatz C, Beran J, Schmid ML, Schulze M, Lopez-Velez R, Fleischer K, Kapaun A, McWhinney P, Kern P, Atougia J, Fry G, da Cunha S, Boecken G (2002) 
Imported Falciparum malaria in Europe: sentinel surveillance data from the European network on surveillance of imported infectious diseases. Clin Infect Dis 34:572-576

15. Wiese L, Bruun B, Baek L, Friis-Møller A, Gahrn-Hansen B, Hansen J, Heltberg O, Højbjerg T, Hornstrup MK, Kvinesdal B, Gomme G, Kurtzhals JA (2006) Bedside diagnosis of imported malaria using the Binax Now malaria antigen detection test. Scand J Infect Dis 38:1063-1068

16. Wongsrichanalai C, Barcus MJ, Muth S, Sutamihardja A, Wernsdorfer WH (2007) A review of malaria diagnostic tools: microscopy and rapid diagnostic test (RDT). Am J Trop Med Hyg 77:119-127

17. World Health Organization. Regional Office for the Western Pacific (2003) Malaria rapid diagnosis: making it work. Meeting report 20-23 January 2003. Manila, the Philippines. Available online at: http://www.searo.who.int/LinkFiles/Malaria_MalariaRDT.pdf. Accessed 19 June 2009

18. De Monbrison F, Gérome P, Chaulet JF, Wallon M, Picot S, Peyron F (2004) Comparative diagnostic performance of two commercial rapid tests for malaria in a non-endemic area. Eur $\mathbf{J}$ Clin Microbiol Infect Dis 23:784-786

19. Grobusch MP, Hänscheid T, Göbels K, Slevogt H, Zoller T, Rögler G, Teichmann D (2003) Sensitivity of $P$. vivax rapid antigen detection tests and possible implications for self-diagnostic use. Travel Med Infect Dis 1:119-122

20. Marx A, Pewsner D, Egger M, Nüesch R, Bucher HC, Genton B, Hatz C, Jüni P (2005) Meta-analysis: accuracy of rapid tests for malaria in travelers returning from endemic areas. Ann Intern Med 142:836-846

21. Richardson DC, Ciach M, Zhong KJ, Crandall I, Kain KC (2002) Evaluation of the Makromed dipstick assay versus PCR for diagnosis of Plasmodium falciparum malaria in returned travelers. $\mathrm{J}$ Clin Microbiol 40:4528-4530
22. Mason DP, Kawamoto F, Lin K, Laoboonchai A, Wongsrichanalai C (2002) A comparison of two rapid field immunochromatographic tests to expert microscopy in the diagnosis of malaria. Acta Trop 82:51-59

23. Pieroni P, Mills CD, Ohrt C, Harrington MA, Kain KC (1998) Comparison of the ParaSight ${ }^{\mathrm{TM}}-\mathrm{F}$ test and the ICT Malaria $\mathrm{Pf}^{\mathrm{TM}}$ test with the polymerase chain reaction for the diagnosis of Plasmodium falciparum malaria in travellers. Trans R Soc Trop Med Hyg 92:166-169

24. Craig MH, Bredenkamp BL, Williams CH, Rossouw EJ, Kelly VJ, Kleinschmidt I, Martineau A, Henry GF (2002) Field and laboratory comparative evaluation of ten rapid malaria diagnostic tests. Trans R Soc Trop Med Hyg 96:258-265

25. Harvey SA, Jennings L, Chinyama M, Masaninga F, Mulholland K, Bell DR (2008) Improving community health worker use of malaria rapid diagnostic tests in Zambia: package instructions, job aid and job aid-plus-training. Malar J 7:160

26. Mayxay M, Newton PN, Yeung S, Pongvongsa T, Phompida S, Phetsouvanh R, White NJ (2004) Short communication: an assessment of the use of malaria rapid tests by village health volunteers in rural Laos. Trop Med Int Health 9:325-329

27. McMorrow ML, Masanja MI, Abdulla SM, Kahigwa E, Kachur SP (2008) Challenges in routine implementation and quality control of rapid diagnostic tests for malaria-Rufiji District, Tanzania. Am J Trop Med Hyg 79:385-390

28. Rennie W, Phetsouvanh R, Lupisan S, Vanisaveth V, Hongvanthong B, Phompida S, Alday P, Fulache M, Lumagui R, Jorgensen P, Bell D, Harvey S (2007) Minimising human error in malaria rapid diagnosis: clarity of written instructions and health worker performance. Trans R Soc Trop Med Hyg 101:9-18

29. Luchavez J, Lintag ME, Coll-Black M, Baik F, Bell D (2007) An assessment of various blood collection and transfer methods used for malaria rapid diagnostic tests. Malar J 6:149 